\title{
вмJ Global Health Spatial and epidemiological drivers of Plasmodium falciparum malaria among adults in the Democratic Republic of the Congo
}

Molly Deutsch-Feldman (D , ${ }^{1}$ Nicholas F Brazeau, ${ }^{1}$ Jonathan B Parr, ${ }^{2}$ Kyaw L Thwai, ${ }^{1}$ Jeremie Muwonga, ${ }^{3}$ Melchior Kashamuka, ${ }^{4}$ Antoinette Tshefu Kitoto, ${ }^{4}$ Ozkan Aydemir, ${ }^{5}$ Jeffrey A Bailey, ${ }^{5}$ Jessie K Edwards, ${ }^{1}$ Robert Verity, ${ }^{6}$ Michael Emch, ${ }^{7}$ Emily W Gower, ${ }^{1}$ Jonathan J Juliano, ${ }^{1,2,8}$ Steven R Meshnick ${ }^{1}$

To cite: Deutsch-Feldman M, Brazeau NF, Parr JB, et al. Spatial and epidemiological drivers of Plasmodium falciparum malaria among adults in the Democratic Republic of the Congo. BMJ Global Health 2020;5:e002316. doi:10.1136/ bmjgh-2020-002316

Handling editor Valery Ridde

- Additional material is published online only. To view please visit the journal online (http://dx.doi.org/10.1136/ bmjgh-2020-002316).

Received 13 January 2020 Revised 22 April 2020 Accepted 25 April 2020

Check for updates

(c) Author(s) (or their employer(s)) 2020. Re-use permitted under CC BY-NC. No commercial re-use. See rights and permissions. Published by BMJ.

For numbered affiliations see end of article.

Correspondence to Molly Deutsch-Feldman; mollydf1@live.unc.edu

\section{ABSTRACT}

Background Adults are frequently infected with malaria and may serve as a reservoir for further transmission, yet we know relatively little about risk factors for adult infections. In this study, we assessed malaria risk factors among adults using samples from the nationally representative, cross-sectional 2013-2014 Demographic and Health Survey (DHS) conducted in the Democratic Republic of the Congo (DRC). We further explored differences in risk factors by urbanicity.

Methods Plasmodium falciparum infection was determined by PCR. Covariates were drawn from the DHS to model individual, community and environmentallevel risk factors for infection. Additionally, we used deep sequencing data to estimate the community-level proportions of drug-resistant infections and included these estimates as potential risk factors. All identified factors were assessed for differences in associations by urbanicity. Results A total of 16126 adults were included. Overall prevalence of malaria was $30.3 \%$ (SE=1.1) by PCR; provincelevel prevalence ranged from $6.7 \%$ to $58.3 \%$. Only $17 \%$ of individuals lived in households with at least one bed-net for every two people, as recommended by the WHO. Protective factors included increasing within-household bed-net coverage (Prevalence Ratio $=0.85,95 \% \mathrm{Cl}=0.76-0.95$ ) and modern housing ( $\mathrm{PR}=0.58,95 \% \mathrm{Cl}=0.49-0.69)$. Communitylevel protective factors included increased median wealth ( $\mathrm{PR}=0.87,95 \% \mathrm{Cl}=0.83-0.92)$. Education, wealth, and modern housing showed protective associations in cities but not in rural areas.

Conclusions The DRC continues to suffer from a high burden of malaria; interventions that target high-risk groups and sustained investment in malaria control are sorely needed. Areas of high prevalence should be prioritised for interventions to target the largest reservoirs for further transmission.

\section{INTRODUCTION}

Malaria remains an important cause of morbidity and mortality in the Democratic

\section{Key questions}

What is already known?

- Malaria transmission is high in the Democratic Republic of the Congo (DRC), though adults are understudied and may serve as a reservoir for further transmission.

- Identifying risk factors helps target interventions to high-risk groups.

- Malaria epidemiology differs between urban and rural areas.

What are the new findings?

- Prevalence of Plasmodium falciparum infection among adults remains high, approximately $30 \%$ with high spatial heterogeneity.

- Risk factors differed between urban and rural areas.

What do the new findings imply?

- Further investment in malaria control programmes in the DRC is needed.

- Malaria control efforts must be tailored to urban versus rural areas to reach high-risk populations.

Republic of the Congo (DRC), which is home to $12 \%$ of all cases globally. ${ }^{1}$ Understanding malaria transmission in the DRC is critical for furthering efforts to eliminate malaria in sub-Saharan Africa. In addition to the high disease burden, there is evidence that the DRC acts as a bridge of transmission, connecting parasites from East and West Africa. ${ }^{2-4}$ To combat transmission and reduce disease burden, it is important to determine risk factors for infection. These factors can be used to identify individuals or groups more likely to become infected and, therefore, more likely to benefit from programmatic interventions. Adult infections remain understudied, as most malaria deaths occur among 
children. ${ }^{56}$ However, adults are frequently infected, often asymptomatically, and may serve as a reservoir for transmission. ${ }^{78}$ Yet, we know very little about risk factors for infection in adults. In this study, we assessed malaria risk factors among adults using data from over 16000 participants from the nationally representative 2013-2014 Demographic and Health Survey (DHS) conducted in the DRC, the largest and most recent health survey conducted in the country.

A small number of studies performed in the DRC have identified important risk factors for infection. ${ }^{410}$ These studies showed that increasing age, wealth and individual bed-net use are protective, as are increasing community-level bed-net use, lower average temperature and higher altitude. ${ }^{9-12}$ However, these studies included only children under the age of five, ${ }^{910}$ or were small and geographically limited. ${ }^{11}{ }^{12}$ A study of adults in the DRC conducted using data from the 2007 DHS identified several risk factors, such as younger age, male sex and lower individual and community-level wealth ${ }^{13}$; however, no nationally representative risk factor studies of adults have been conducted since.

In this study, we evaluated current individual, household, community and environmental risk factors for malaria among adults. Additionally, because increasing antimalarial drug resistance is a growing concern in the DRC, we sought to understand the relationship between community-level resistance and infection prevalence. ${ }^{14}{ }^{15}$ Similarly, the association between increasing antimalarial use and malaria prevalence among adults has not been studied in the DRC. Understanding the role of these factors is critical for determining drivers of malaria infection. The different scales of the factors demonstrate the various levels at which malaria interventions can be targeted.

This study builds on previous work in the DRC by evaluating the role of urbanisation on risk factors for infection. Understanding the effect of urbanisation on malaria transmission is a critical part of intervention planning. ${ }^{16-18}$ Unlike other infectious diseases, which thrive in cities due to increased population density, malaria transmission is often lower in urban areas as compared with rural areas. ${ }^{19}{ }^{20}$ This is due to several factors, including reduced vector populations, lower biting rates and better access to therapeutics. ${ }^{19}$ As a result, the effects of malaria control programmes have been shown to differ between cities and rural areas. Multiple studies conducted in Nigeria and Benin found that bed-net ownership and use were higher among rural populations than urban. ${ }^{21-23}$ Past work conducted in Liberia determined that the effects of bed-nets differed between the settings, with increasing community-level bed-net coverage being protective in urban areas but displaying no effect in rural areas. ${ }^{24}$ Additionally, the adaptation of Anopheles species to urban settings indicates the need to further explore the role of urbanisation on malaria risk. ${ }^{25}$ We explored this relationship in the DRC by assessing whether the effects of various risk factors for infection differed between individuals in urban vers rural areas.
The findings from this study can be used to identify individuals and communities at higher risk for malaria infection in the DRC. They also shed light on differences in the epidemiology of malaria between urban and rural settings.

\section{METHODS}

\section{Study population}

The data from this study are drawn from the 2013 to 2014 DHS conducted in the DRC. The DHS Program, run by the United States Agency for International Development in conjunction with local governments, carries out periodic cross-sectional surveys in over 90 countries. ${ }^{26}$ In the DRC, the DHS was conducted by the coordinated efforts of several governmental ministries. Using a nationally representative randomised cluster sampling method, sampling cluster sites were first selected from a map of enumerated areas across the country with the probability of selection proportional to the size (number of households) of the cluster. ${ }^{27}$ Next, households were randomly selected for inclusion from within clusters. ${ }^{28}$ Cluster sizes differed between urban and rural areas with approximately $5-15$ more households included in rural areas. ${ }^{28}$ To account for this nested cluster sampling strategy when analysing the data, each cluster was assigned a sampling weight. DHS survey conductors visited selected households, obtained consent and administered an extensive questionnaire covering a broad range of topics including nutrition, education, health history and infectious diseases status. ${ }^{27}$ Blood samples were collected on filter paper for HIV testing; a second blood spot was also shipped to The University of North Carolina for PCR testing of malaria.

A total of 18257 adults from 12549 households were included and asked to provide blood samples. This included 9601 women (aged 15-49) and 8656 men (aged 15-59). In addition, 9790 children (aged 6 months to 5 years) were part of the survey. Although they were not included in this analysis, they have been examined previously. ${ }^{9} 1429$

\section{Risk factor selection}

Potential risk factors of Plasmodium falciparum infection were determined by consulting previous studies and based on biological plausibility. We selected individual, household and cluster (community) level risk factors that have demonstrated associations with malaria risk. ${ }^{9} 1013$ Data regarding these factors were drawn from the DHS questionnaire. Individual factors included age, biological sex, HIV infection status, education and wealth index. The DHS Program calculates wealth index as a composite variable based on each household's assets and housing materials $^{30}$. Using principal components analysis (PCA), participants were then grouped into wealth quintiles: poorest, poor, middle, rich and richest. ${ }^{30}$ We also assessed bed-net use, which was determined by whether the individual reported sleeping under a long-lasting insecticide net (LLIN) the previous night. At the household level, we constructed a 'net ratio' variable by dividing the number of total nets per household by the number of individuals 
within the household. This was then dichotomised into ratios of $<0.5$ versus those of 0.5 or higher (ie, at least one net for every two household members). We also created a composite 'housing materials' variable, dichotomised as either traditional or modern, based on data from the roof, wall and floor material variables (further details are available in the online supplementary text). ${ }^{31} \mathrm{~A}$ recent study from The Gambia demonstrated that metal roofs were protective against malaria infection, ${ }^{32}$ thus we also assessed the relationship between malaria and metal roofing alone, separate from modern housing. Clusterlevel factors included proportion reporting LLIN use, median wealth index and median education level. We also assessed average annual precipitation and temperature as well as the range in temperature (defined as the difference between the average temperature in January and average temperature in July) and the cluster vegetation index. ${ }^{33}$ Data regarding these factors were drawn from the DHS Program, which collects a range of environmental and geological data. ${ }^{33}$

\section{PCR diagnosis}

The primary outcome for this study was P. falciparum malaria infection as determined by real-time PCR. In this manuscript, malaria is defined as infection with $P$. falciparum parasites. PCR was performed on the blood spots collected as part of the DHS survey. Whole blood collected by finger prick was spotted onto Whatman filter paper, dried at ambient temperature and initially stored with dessicant at $-80^{\circ} \mathrm{C}$ in Kinshasa until punching (one $6 \mathrm{~mm}$ punch per subject) and shipment to the University of North Carolina (UNC) at Chapel Hill. DNA was extracted from the blood spots using a Chelex extraction assay and used for PCR testing. ${ }^{34}$ The PCR assay detects the $P$. falciparum lactate dehydrogenase gene $(p f l d h)$ with a limit of detection of $5-10$ parasites/mL. ${ }^{35} 36$ The human beta-tubulin (HumTuBB) gene was used as a positive control, and any samples that failed to amplify HumTuBB were excluded. The duplexed $p f l d h$ and HumTuBB quantitative PCR assay was performed using reaction conditions, primers and quality control measures for highthroughput PCR exactly as previously described. ${ }^{37}$ All samples were run in duplicate. Samples that amplified pfldh in only a single replicate were considered negative if the cycle threshold value was higher than 38. All laboratory assays were completed at UNC

\section{Genetic analyses}

Community-level drug resistance was determined using second-generation sequencing data obtained using molecular inversion probes (MIPs) that target known molecular markers of resistance to antimalarial drugs. ${ }^{14}$ MIPs are a technology for obtaining highly multiplexed deep sequencing data that have recently been applied to Plasmodia species. $^{14}$ Using previously generated sequencing data from 1065 children living in 333 clusters enrolled in the 2013-2014 DRC DHS, ${ }^{1415}$ we assessed single nucleotide polymorphisms (SNPs) of the pfdhps
(A437G, K540E and A581G) and pfcrt (K76T) genes known to be associated with sulfadoxine-pyramethamine (SP) and chloroquine resistance, respectively. ${ }^{148-40}$ We used these individual-level data to estimate cluster-level prevalence for each SNP for the 489 DHS clusters with geolocation data using the PrevMap package in R, which fits a spatial model using a Gaussian process. ${ }^{41}$ We fit the model to generate estimates of the underlying allele frequency distribution using maximum likelihood and running 10000 simulations. Further model details are available in the online supplementary text.

\section{Urban/rural classification}

Potential misclassification of urbanicity is a concern in large surveys with complex sampling frames. ${ }^{42}$ To minimise such misclassification, we conducted a PCA incorporating variables with a demonstrated relationship to urbanicity. ${ }^{334344}$ These variables were degree of built environment, night-time lights, total population as of 2014, population density as of 2014 and estimated travel time to the nearest city. The DHS collects these data from a variety of remote sensing and modelling databases including WorldPop, the Joint Research Centre and the Climate Research Unit at the University of East Anglia. ${ }^{33} 45-47$ The DHS collects these variables for each cluster; all variables were scaled and log transformed for the analysis. From the PCA, we extracted the values for the first principal component (PC) for each cluster, and generated a new urban/rural variable by dichotomising the PC1 value at the $75 \%$ percentile (eg, urbanicity is represented by the upper quartile of PC1). The new measure of urbanicity was used to assess differences in risk factors between urban and rural areas. We compared the classifications generated for this analysis to the urban/rural categorisations from the DHS using Pearson's correlation coefficient.

\section{Modelling and statistical analyses}

All analyses were performed using the R statistical platform V.3.5.2. ${ }^{48}$ We assessed the relationship between each risk factor and PCR detectable malaria infection using bivariate log-binomial regression models (ie, not adjusted for other variables) to estimate prevalence ratios. We fit models incorporating sampling weights using the survey package in $\mathrm{R}$, which uses weighted generalised estimating equations to properly account for the multi-level sampling scheme and correlation between individuals in the same sampling cluster. ${ }^{49}{ }^{50}$ This allowed us to assess individual and cluster-level factors simultaneously. As most households had only one adult member, we did not need to account for within-household correlation. We used bivariate models as the aim was to assess marginal associations, not causal relationships. ${ }^{51}$ Each identified risk factor was then further assessed to determine if the association with malaria prevalence differed by urban/rural status by including the urban variable and an interaction term between each factor and the urban variable in the model. In general, variables were included in the model in the 
form that the data were collected (ie, categorical variables kept as categorical), though some continuous variables were scaled and proportions were logit transformed. As done previously for MIP genetic data, we generated spatially smoothed prevalence and SE estimates using the PrevMap package (online supplementary text). ${ }^{41}$

In addition to the risk factor modelling we conducted several subsequent analyses. As the DHS asks multiple questions regarding bed-net use, we conducted a sensitivity analysis to compare various coding methods for net use. Further details are presented in the online supplementary text. We also assessed whether the association between individual bed-net use and malaria risk differed across categories of overall cluster malaria prevalence. For this analysis, we used previously published data from children included in the 2013-2014 DHS to determine community-level prevalence in order to avoid including the outcome data of adult infections from 2013 in determining cluster-level prevalences. ${ }^{9} 29$

\section{Patient and public involvement}

This research was conducted without patient involvement

\section{RESULTS}

Our analysis included 16126 adults from the original 18257 initially selected for inclusion in the DHS. Individuals were sampled from 533 geographically dispersed clusters; GPS data were missing for 44 clusters, resulting in 489 clusters for analysis (figure 1). Characteristics describing the individuals with missing GPS data are available in online supplementary table 2. After final data processing, 16363 individuals had complete PCR and DHS covariate data. Two-hundred and thirty-seven individuals were not considered 'de jure' (members of the sampled household, rather than visitors who slept in

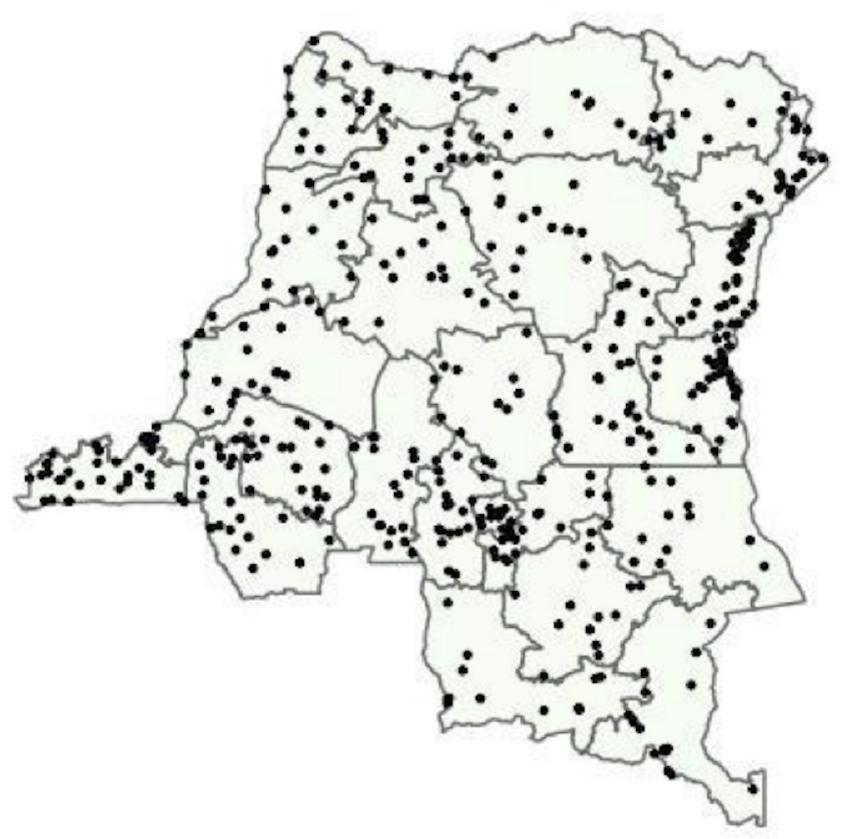

Figure 1 Sites of 2013-2014 DHS sampling clusters.

18,257 bloodspots

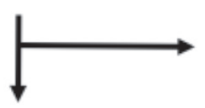

289 duplicates, no barcodes, etc.

17,968 PCR reactions

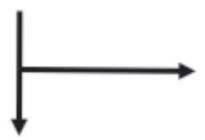

203 barcode errors or no beta-tubulin amplification, 1402 missing GPS data

16,363 matched with DHS metadata

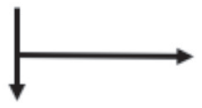

237 non-"de jure" individuals

\section{6,126 individuals} included in analysis

Figure 2 Flowchart of samples included in analysis.

the household the previous night ${ }^{52}$ and, thus, were not included in the analysis), resulting in a final data set of 16126 individuals (figure 2). Overall PCR prevalence of P. falciparum infection was $30.3 \% \quad(\mathrm{SE}=1.1)$. The results of the PrevMap analysis demonstrated the high spatial heterogeneity of infection; community prevalence estimates ranged from $0 \%$ to $76 \%$ (figure 3 ). The map of the model SEs indicated low variance in the estimates across the DRC (online supplementary figure S1). Prevalence estimates by province ranged from $6.7 \%$ in Nord-Kivu to $58.3 \%$ in Bas-Uele (online supplementary table 1).

Reported bed-net use was low; though $75 \%$ of adults reported owning a LLIN, only $54 \%$ reported sleeping under a net the previous night. The average ratio of nets per household member was 0.27 , indicating approximately one net per four household members. Only $17 \%$ of adults lived in houses with at least one net per two household members; $24 \%$ of all households had at least one net for every two people. Baseline characteristics of study participants are presented in table 1 .

\section{Risk factor analysis}

Several covariates were associated with prevalence of malaria infection (table 2). Individual protective factors included increasing age, female sex, increasing education and increasing wealth index. Protective household factors included living in a house made of modern housing materials and having a metal roof. At the cluster level, increasing median wealth index was protective. Higher use of SP among pregnant women at the cluster level was also found to be associated with lower prevalence of malaria. Drug-resistance mutations were more common in lowprevalence clusters. Increasing average temperature was 


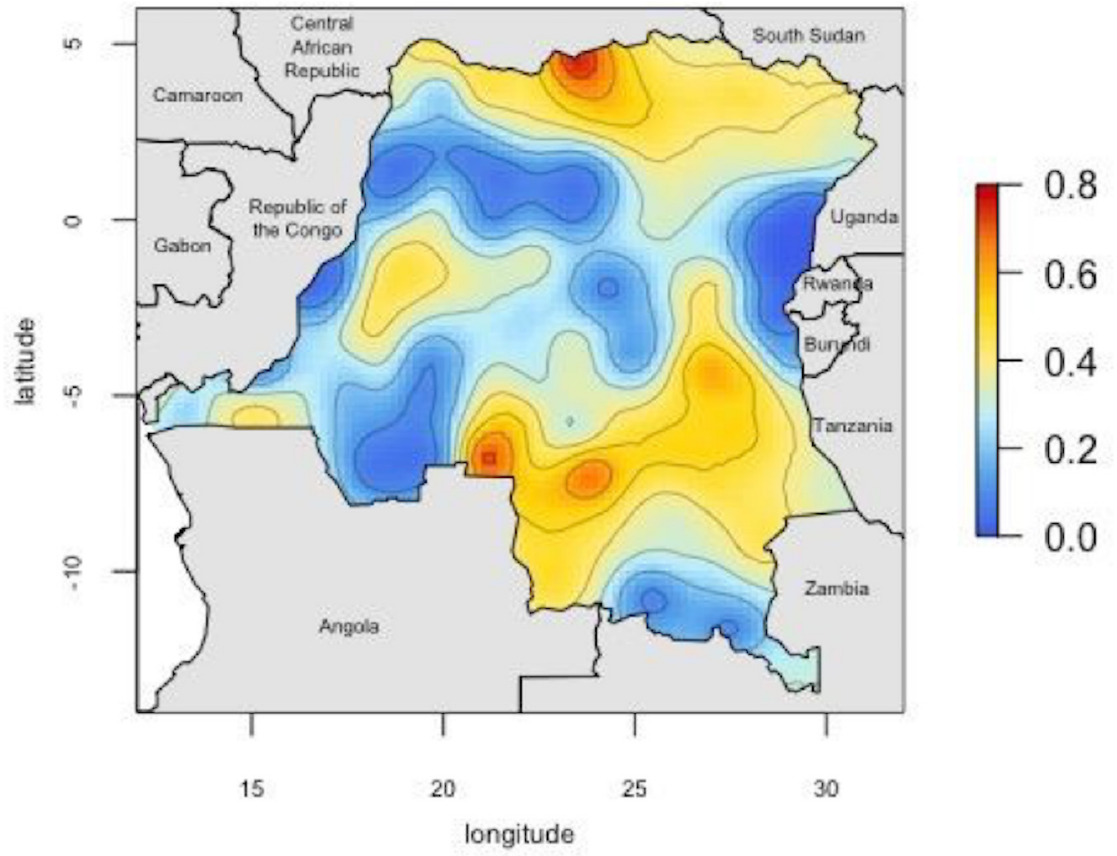

Figure 3 Map of predicted P. falciparum PCR prevalence estimates. Smoothed prevalence estimates, incorporating the sampling weights, were generated using the PrevMap package in $\mathrm{R}^{39}$. Predicted proportions range from 0-0.76.

associated with increased prevalence of infection as was increasing vegetation. A larger range in average monthly temperature was associated with lower malaria prevalence. Full risk factor modelling results are available in table 2.

We found a protective association of individual LLIN use and no effect of increasing community LLIN use, in contrast to previous findings in the DRC. ${ }^{9}$ At the household level, a net ratio greater than 0.5 (ie, at least one net per two household individuals) was protective, compared with households with a lower ratio. When we stratified individual net use by insecticide type, we observed a protective effect of deltamethrin-treated nets and a signal for protection with alphacypermethrin nets. There was no significant protective effect of permethrin-treated nets, a finding that has been previously reported for children in the DRC. ${ }^{9}$ Restricting LLIN use to only those who had reported using a net treated with deltamethrin or alphacypermethrin, we found a protective effect of increasing community-level net use. The sensitivity analysis of net use showed no differences between measurement options or the coding choice for net use (online supplementary figure 1). Additionally, the sensitivity analysis showed no difference in the association when restricting 'net-use' to only nets under 3 years old. A comparison of individual LLIN net use between adults and children included in the 2013-2014 DHS indicated similar protective effects (online supplementary table 4 ).

\section{Analysis of associations by Urban/rural status}

There were several socioeconomic-related risk factors whose association varied by urban/rural status (figure 4 ). These included individual-level wealth, with increasing wealth showing a protective effect in urban areas but not rural. A similar trend was observed for increasing individual-level education. Additionally, both modern housing and metal roofs were protective in urban areas but not in rural areas. Cluster-level wealth demonstrated problematically high collinearity with urbanicity and thus could not be modelled. Full numeric results from this analysis are presented in online supplementary table 3 .

Overall, urbanicity did not impact the association between LLIN use and malaria prevalence, either at the individual or cluster level. However, when we subset LLIN use to only deltamethrin and alphacypermethrin nets, the only insecticides that demonstrated a protective effect in the initial analyses, we observed that increasing individual and community use of these nets were more protective in rural areas than in urban areas. Similarly, the analysis of LLIN use by cluster-level prevalence found that individual net use was more protective in areas of higher overall malaria prevalence (figure 5 ).

The correlation between the urban/rural categories generated for this analysis and the original DHS categories was 0.75 .

\section{DISCUSSION}

In this study, utilising data from the largest and most recent nationally representative health survey conducted in the DRC, we found a high national prevalence of $P$. falciparum malaria $(30 \%)$ among adults. We also found high spatial heterogeneity. Community-level prevalence estimates reached as high as $76 \%$. Malaria is often considered a paediatric disease as the majority of deaths occur among children. ${ }^{5}$ However, our findings emphasise that the burden of disease among adults is high in the DRC and highlight the importance of adult infections in malaria epidemiology. Addressing adult infections 
BMJ Global Health

Table 1 Descriptive statistics of the study population by Plasmodium falciparum PCR status

\begin{tabular}{|c|c|c|c|}
\hline & PCR positive & PCR negative & Total \\
\hline Unweighted total number* & 5372 & 10754 & 16126 \\
\hline Weighted proportion $†$ & $30.3 \%$ & $69.7 \%$ & \\
\hline \multicolumn{4}{|l|}{ Individual level $†$} \\
\hline Median age (IQR) & $26(19-36)$ & $29(21-38)$ & $28(20-38)$ \\
\hline Number of females (\%) & $2360(47.6)$ & $6202(54.4)$ & $8562(52.3)$ \\
\hline Number of HIV positive (\%) & $30(0.6)$ & $126(1.1)$ & $156(0.9)$ \\
\hline \multicolumn{4}{|l|}{ Education category (\%) } \\
\hline No school & $515(10.4)$ & $1140(10.0)$ & $1655(10.1)$ \\
\hline Primary school & $1618(32.6)$ & $3346(29.4)$ & $4964(30.4)$ \\
\hline Secondary school & $2662(53.8)$ & $6079(53.4)$ & $8741(53.5)$ \\
\hline Higher than secondary & $156(3.2)$ & $826(7.2)$ & $982(6.0)$ \\
\hline Owns a bed-net (\%) & $3615(72.9)$ & $8589(75.3)$ & $12204(74.6)$ \\
\hline Slept under bed-net previous night (\%) & $2462(49.7)$ & $6422(56.3)$ & $8884(54.3)$ \\
\hline \multicolumn{4}{|l|}{ Wealth category (\%) } \\
\hline Poorest & $1059(21.4)$ & $1840(16.1)$ & $2899(17.7)$ \\
\hline Poor & $1030(20.8)$ & $2014(17.7)$ & $3044(18.6)$ \\
\hline Middle & $1122(22.6)$ & $2124(18.6)$ & $3246(19.8)$ \\
\hline Rich & $1041(21.0)$ & $2249(19.7)$ & $3290(20.1)$ \\
\hline Richest & $704(14.2)$ & $3178(27.9)$ & $3882(23.7)$ \\
\hline \multicolumn{4}{|l|}{ Household level } \\
\hline Average number of bed-nets per person (SE) & $0.25(0.007)$ & $0.27(0.008)$ & $0.27(0.007)$ \\
\hline Modern housing (\%) & $593(12.0)$ & $2514(22.1)$ & $3107(19.0)$ \\
\hline Metal roofing (\%) & $1564(31.6)$ & $5147(45.1)$ & $6711(41.0)$ \\
\hline \multicolumn{4}{|l|}{ Cluster level $†$} \\
\hline Median age (IQR) & $30.0(28.5-31.3)$ & $29.7(28.2-31.0)$ & $30.0(28.6-31.6)$ \\
\hline Urban (\%) & $1329(26.8)$ & $4297(37.7)$ & $5626(34.4)$ \\
\hline Median education (IQR) & $2(1-2)$ & $2(1-2)$ & $3(2-3)$ \\
\hline Median wealth score (IQR) & $3(2-4)$ & $3(2-5)$ & $3(2-4)$ \\
\hline Average annual centimetres of precipitation (SE) & $152.2(1.4)$ & $149.9(1.7)$ & $150.6(1.4)$ \\
\hline Average temperature (SE) & $24.7(0.1)$ & $23.7(0.2)$ & $24.0(0.2)$ \\
\hline Vegetation index (SE)‡ & $3934.5(52.6)$ & $3660.7(69.5)$ & $3734.6(58.7)$ \\
\hline \multicolumn{4}{|l|}{ \% Drug resistance§ } \\
\hline Any pfdhps & $92.2(0.7)$ & $95.1(0.4)$ & $94.2(0.5)$ \\
\hline pfdhps K540E & $23.5(1.7)$ & $32.9(2.5)$ & $30.1(2.1)$ \\
\hline pfdhps A581G & $1.8(0.1)$ & $3.9(0.4)$ & $3.2(0.3)$ \\
\hline pfcrt K76T & $56.0(2.3)$ & $64.8(1.9)$ & $62.1(1.8)$ \\
\hline \% Net ownership (SE) & $73.7(1.3)$ & $75.0(1.5)$ & $74.6(1.3)$ \\
\hline \% Net usage (SE) & $52.7(1.4)$ & $54.6(1.6)$ & $54.0(1.4)$ \\
\hline \% SP use among pregnant women (SE) & $25.3(1.3)$ & $28.0(1.1)$ & $27.2(1.1)$ \\
\hline
\end{tabular}

${ }^{*}$ These are unweighted raw numbers and do not represent the sum of the subsequent values in the table as subsequent values incorporate sample weights.

†Proportions and numbers with sampling weights applied.

$\neq$ Vegetation index ranges from 0 (least vegetation) to 10000 (most vegetation). ${ }^{33}$

$\S$ Estimates generated using previously published data. ${ }^{1415}$

SP, sulfadoxine-pyramethamine.

is critical for eliminating malaria as adults may serve as a reservoir for further transmission of parasites. ${ }^{8}$ The high level of spatial heterogeneity across the country underscores the need to study malaria on a subnational scale and develop targeted strategies for areas of highest prevalence (and thus a larger reservoir). The spatial 
Table 2 Risk factor analysis results

\begin{tabular}{|c|c|c|c|}
\hline Variable & Prevalence Ratio & $95 \% \mathrm{CI}$ & P value* \\
\hline \multicolumn{4}{|l|}{ Individual level } \\
\hline Age (scaled) & 0.86 & 0.83 to 0.89 & $<0.001$ \\
\hline Female sex & 0.83 & 0.78 to 0.88 & $<0.001$ \\
\hline HIV positive & 0.63 & 0.38 to 1.03 & 0.067 \\
\hline \multicolumn{4}{|l|}{ Education category: } \\
\hline No school (REF) & -- & -- & -- \\
\hline Primary school & 1.05 & 0.92 to 1.18 & 0.473 \\
\hline Secondary school & 0.98 & 0.85 to 1.13 & 0.761 \\
\hline Higher than secondary & 0.51 & 0.38 to 0.70 & $<0.001$ \\
\hline \multicolumn{4}{|l|}{ Wealth category } \\
\hline Poorest (REF) & -- & -- & -- \\
\hline Poor & 0.93 & 0.83 to 1.04 & 0.189 \\
\hline Middle & 0.95 & 0.85 to 1.06 & 0.330 \\
\hline Rich & 0.87 & 0.73 to 1.02 & 0.088 \\
\hline Richest & 0.50 & 0.40 to 0.61 & $<0.001$ \\
\hline Owns a net & 0.92 & 0.82 to 1.03 & 0.138 \\
\hline Slept under LLIN & 0.83 & 0.76 to 0.91 & $<0.001$ \\
\hline \multicolumn{4}{|l|}{ Net type } \\
\hline No net (REF) & -- & -- & -- \\
\hline Permethrin & 0.94 & 0.78 to 1.12 & 0.477 \\
\hline Alphacypermethrin & 0.68 & 0.38 to 1.22 & 0.198 \\
\hline Deltamethrin & 0.81 & 0.73 to 0.91 & $<0.001$ \\
\hline \multicolumn{4}{|l|}{ Household level } \\
\hline Net ratio† & 0.85 & 0.76 to 0.95 & 0.003 \\
\hline Modern housing & 0.58 & 0.49 to 0.69 & $<0.001$ \\
\hline Metal roofing & 0.66 & 0.58 to 0.75 & $<0.001$ \\
\hline \multicolumn{4}{|l|}{ Cluster level } \\
\hline Urban & 0.70 & 0.59 to 0.83 & $<0.001$ \\
\hline LLIN ownershipł & 0.97 & 0.93 to 1.00 & 0.058 \\
\hline LLIN usage & 0.98 & 0.92 to 1.04 & 0.457 \\
\hline $\begin{array}{l}\text { LLIN usage (deltamethrin and alphacypermethrin nets } \\
\text { only) } \ddagger\end{array}$ & 0.95 & 0.90 to 1.00 & 0.040 \\
\hline Education & 0.91 & 0.79 to 1.04 & 0.174 \\
\hline Wealth & 0.87 & 0.83 to 0.92 & $<0.001$ \\
\hline Precipitation (scaled) & 1.06 & 0.97 to 1.17 & 0.179 \\
\hline Temperature (scaled) & 1.32 & 1.23 to 1.42 & $<0.001$ \\
\hline Temperature range (scaled) & 0.91 & 0.85 to 0.98 & $<0.001$ \\
\hline Vegetation index (scaled) & 1.18 & 1.09 to 1.27 & $<0.001$ \\
\hline SP use among pregnant women $\ddagger$ & 0.92 & 0.89 to 0.96 & $<0.001$ \\
\hline \multicolumn{4}{|l|}{ Drug resistance prevalence } \\
\hline Pfdhps A437G & 0.88 & 0.85 to 0.91 & $<0.001$ \\
\hline pfdhps K540E & 0.95 & 0.93 to 0.98 & $<0.001$ \\
\hline pfdhps A581G & 0.89 & 0.85 to 0.94 & $<0.001$ \\
\hline pfcrt K76T & 0.93 & 0.91 to 0.96 & $<0.001$ \\
\hline
\end{tabular}

${ }^{*} \mathrm{P}$ value of the test of the null hypothesis that the Prevalence Ratio=1. Values below 0.05 are bolded.

†Ratio $\geq 0.5$ vs $<0.5$.

†Logit transformed.

LLIN, long-lasting insecticide net; SP, sulfadoxine-pyrimethamine. 


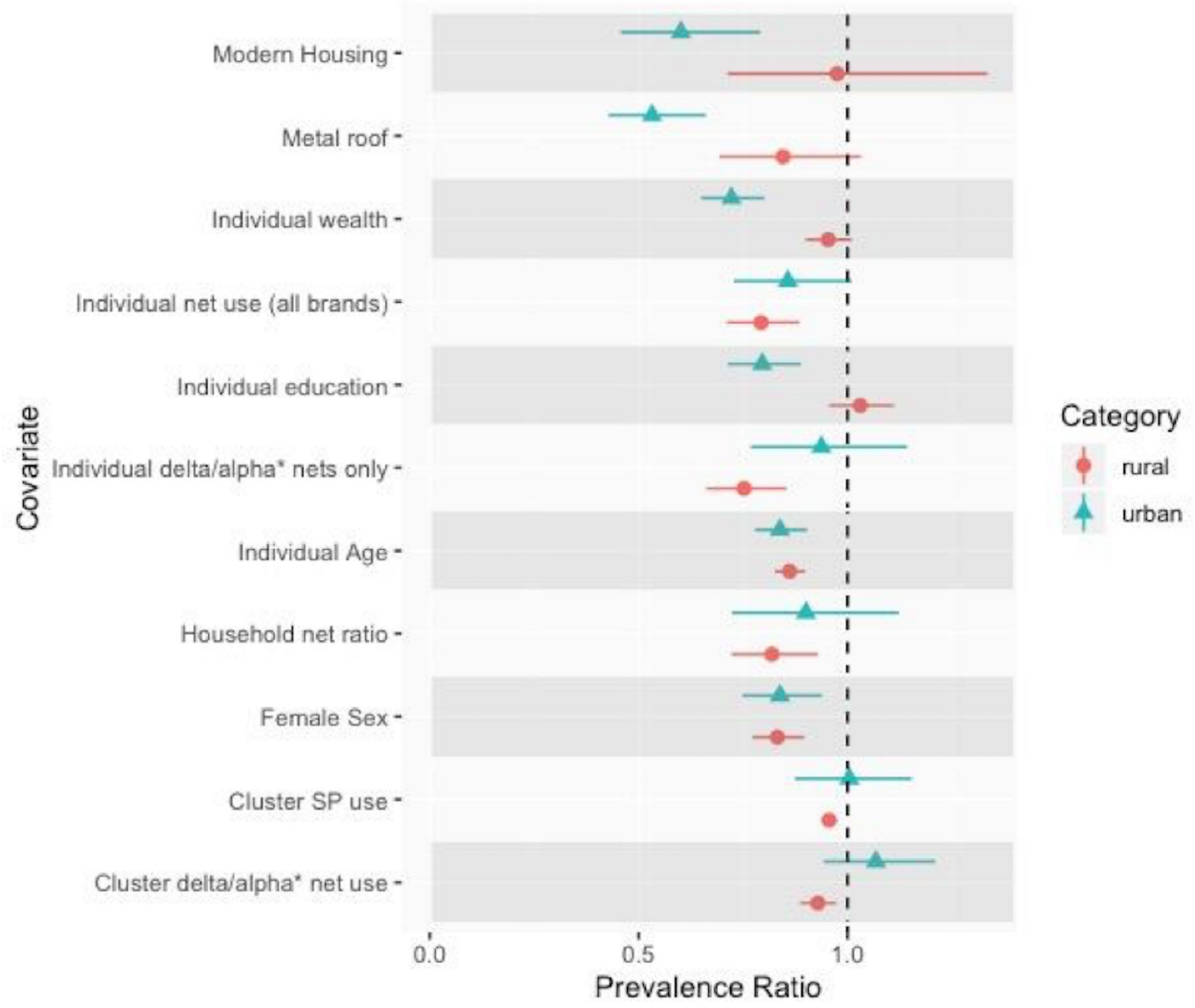

Figure 4 Results of the analysis comparing risk factor effects between urban versus rural areas. Prevalence ratios and confidence intervals by urban status are presented for each risk factor. Urban results are presented with blue triangles, rural results with red circles. Differences in point estimates indicate differences of the prevalence ratio by urbanicity. The associations of several factors, such as modern housing, education, and wealth, demonstrated differences between urban and rural areas. The null value (Prevalence Ratio $=1$ ) is indicated with a vertical dashed line.

distribution of infections among adults matches that of infections among children included in the 2013-2014 DHS, indicating similar underlying spatial processes. ${ }^{53}$ Similarly, the prevalence map displays a similar pattern to that from adults included in the 2007 DHS. ${ }^{13}$ The areas of high versus low prevalence are likely driven by several factors such as altitude and urbanicity. The mountainous regions of Kivu in the northeast have very little malaria, while the mining districts in southern DRC have higher prevalence.

The national prevalence estimate of 30\% among adults is similar to that from the 2007 DHS, suggesting a need for increased investment in malaria control in the DRC and further understanding of drivers of transmission. ${ }^{13} 54$ We observed that the prevalence is unchanged from 2007 despite increases in LLIN coverage and the number of individuals being tested and treated for infection. ${ }^{54}$ Thus, understanding malaria risk factors in all age groups remains critical for the design and implementation of effective interventions. Several risk factors identified in an analysis of the 2007 DHS data were also associated with increased infection prevalence in this study, highlighting their continued importance in the epidemiology of malaria. These include younger age, male sex and lower community-level wealth. ${ }^{13}$ The factors identified in this study, many of which have also been identified in other countries, ${ }^{55-57}$ are particularly important for identifying infected individuals in the DRC, as many adult infections are asymptomatic and often go undetected. ${ }^{8}$ Thus, targeting malaria interventions towards younger adults, men and poorer communities could maximise their impact.

In this study, we found that individuals living in households with a bed-net ratio greater than 0.5 , that is, at least one net for every two household members, had lower prevalence of malaria. This ratio is recommended by the $\mathrm{WHO}$ in order to ensure a sufficient number of nets for household members. ${ }^{58}$ Though previous studies have assessed the role of increasing community-level net use, the effect of within household 'net coverage' has not been studied, to our knowledge. Our findings demonstrate the importance of the number of nets within a household and support the WHO recommendation. However, only $24 \%$ of households in 2013-2014 DHS had a net ratio of 0.5 or higher. The 2019 WHO World Malaria Report estimated that currently, approximately $50 \%$ of households in the DRC have at least one net for every two people. ${ }^{1}$ Thus, the DRC is making progress towards achieving the WHO recommendation; however, there is still a lot of work to be done to make sure all households meet the recommendation. It is critical that future bed-net distribution campaigns ensure that enough nets are provided to each household. 


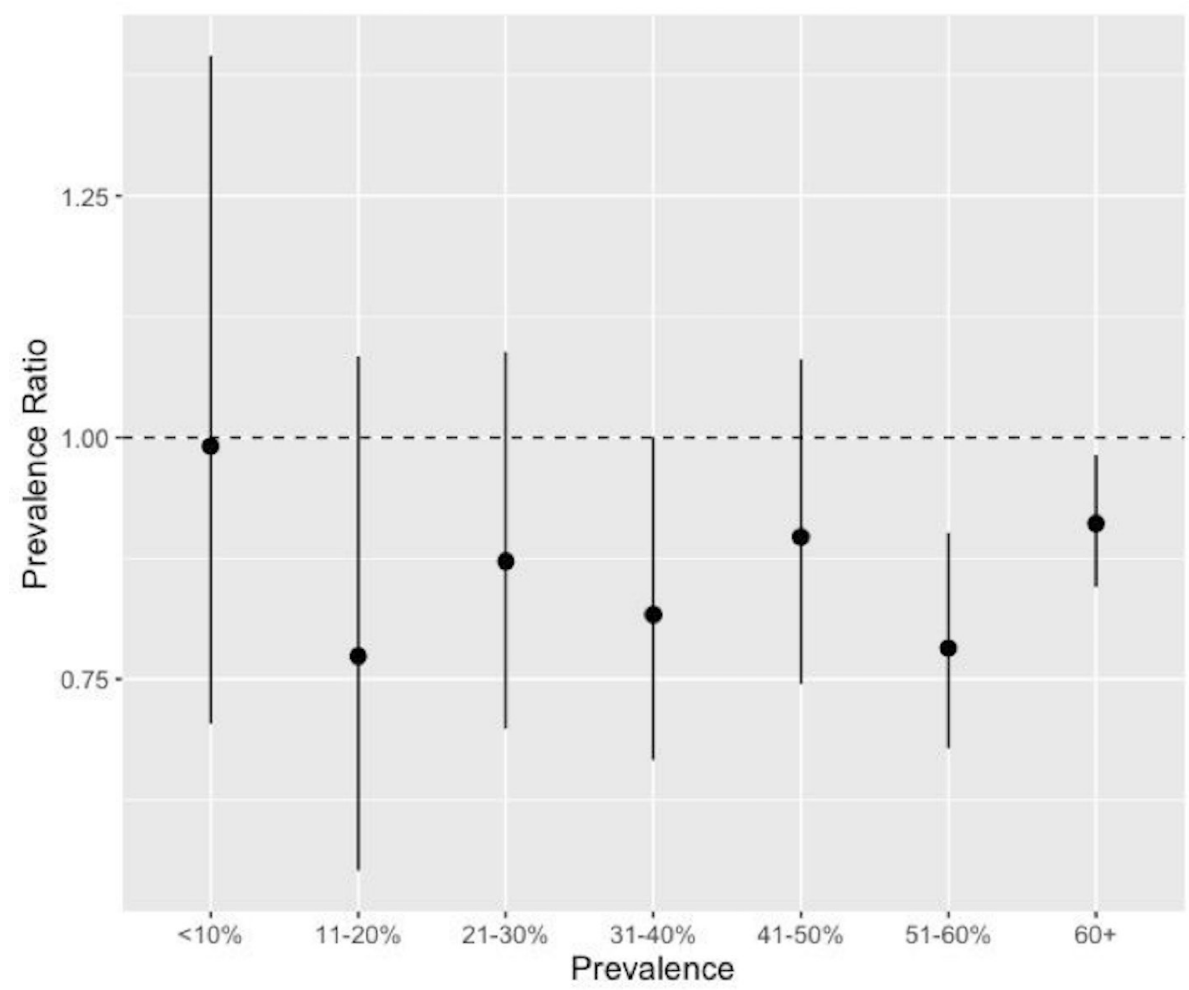

Figure 5 Effect of individual bednet use by cluster-level malaria prevalence. We used previously published data from children included in the 2013-2014 Demographic and Health Survey in order to determine cluster-level prevalence. ${ }^{28}$ Individual bednet use was more protective in clusters with higher malaria prevalence. The null value (Prevalence Ratio $=1$ ) is indicated with a horizontal dashed line.

In this analysis, we did not observe the overall protective effect of community bed-net use that has been previously demonstrated among children in the DRC, though there was a protective effect of increasing community use of deltamethrin and alphacypermethrin-treated nets. ${ }^{9}$ As mentioned, we did see a protective effect of increasing within household net coverage, which may be a more important factor for reducing infection than community net use. Our findings of greater protection from nets in higher prevalence areas and in rural areas are supported by a recent meta-analysis that found increasing community malaria prevalence associated with a greater protective effect of LLIN use $(\mathrm{OR}=0.80) .{ }^{59}$ Thus, bed-net distribution campaigns may be more successful in rural or generally higher prevalence areas than in cities.

Poor LLIN efficacy may be due to several factors such as adult sleeping behaviours and changing mosquito biting patterns, and demonstrates that malaria control programmes cannot rely on LLIN use alone. Many adults go to sleep after Anopheles mosquitoes begin biting, rendering LLINs less useful. ${ }^{60}$ Additionally, recent studies have demonstrated a shift in Anopheles biting hours earlier in the evening as a result of LLIN use. ${ }^{61}$ These findings point to the need to consider additional malaria control programmes such as improved community testing for malaria and targeted mass drug administration. ${ }^{62-64}$

The findings from the genetic analysis of mutations associated with SP and chloroquine resistance indicate that areas of higher prevalence of drug resistance had lower prevalence of malaria infection. While this is a marginal association and does not reflect a causal relationship, this could be due to the amount of antimalarial drug use, leading to lower overall prevalence of malaria but increased resistance through increased selective pressure. Future studies should aim to investigate the causal effect of increasing community-level drug resistance on malaria risk; however, this was not the primary aim of this study. Additionally, cluster-level SNP prevalences were modelled estimates generated using data from children and thus may not be representative of overall communitylevel prevalence.

Modern housing and metal roofing were both associated with lower prevalence of malaria, though the association was more pronounced in urban areas than in rural areas. The effect of metal roofing agrees with findings from a recent study conducted in The Gambia, which found 38\% lower mosquito survival and lower malaria prevalence among villages with higher proportions of metal roofs. ${ }^{32}$ The authors of the study propose that this is due to the higher temperatures of metal roofs during the day, leading to lower mosquito survival. The present findings suggest that housing improvements may help reduce malaria risk, either directly as proposed by the Gambian study, or indirectly through overall improvements in living conditions and socioeconomic status.

This study also highlights differences in the epidemiology of malaria between urban and rural areas, confirming the need to tailor interventions to different 
populations in cities versus rural areas. Increasing wealth and education are both highly protective in urban areas but not in rural areas. This may be a result of increased access to prevention and treatment methods in urban areas, as has been observed in other countries. A study from Equatorial Guinea found individuals in rural areas waited longer to seek treatment for malaria infections compared with those in urban areas, and were more likely to be treated at home rather than in a health facility. ${ }^{65}$ These results also agree with findings from previous studies that poor individuals living in urban areas have similar health risks to the general rural population. ${ }^{66}$ Thus, in urban areas, though individuals overall tend to be wealthier, the malaria-positive population is poorer and less educated than those infected in rural areas. Malaria control programmes should take these differences in high-risk populations into account, targeting poorer or less-educated individuals in cities and ensuring that interventions are accessible for these populations. Conversely, interventions in rural areas need to be more widespread and accessible to a more diverse population.

This study has several strengths. First, it uses nationally representative, population-based data from over 16000 individuals, the largest health survey conducted in the DRC. This allows us to make inferences regarding the country as a whole. Other recent malaria risk factor studies have fewer individuals or are conducted in a smaller geographic area. ${ }^{11}{ }^{12}$ Second, we determined malaria infection using high-throughput real-time PCR, the most sensitive method for diagnosing infection. ${ }^{29}$ Third, it leverages country-wide drug resistance genotyping data to inform community-level epidemiological analysis. Lastly, this study incorporates multiple types of data including survey, molecular, deep sequencing drug resistance and geospatial data collected at the individual, household, community and environmental level. This allowed us to demonstrate the different scales of malaria risk factors; control programmes should aim to intervene at each of these levels.

The findings from this study are subject to limitations. Several of the covariates included in the study were obtained from self-reports, including LLIN usage. While self-reported data are subject to recall bias, the DHS questionnaire asks multiple questions regarding net use that allowed us to assess bias. The sensitivity analysis comparing these questions indicated no difference in modelling results between the questions, providing confidence that bias from self-reports is minimal. Second, this analysis evaluated marginal associations and thus the findings cannot be interpreted as causal effects. However, the associations are useful for identifying and targeting interventions to higher risk individuals and groups such as younger, less-educated men. Additionally, as the DHS is a cross-sectional survey, we did not assess the effects of seasonality. Finally, we could not directly assess the epidemiology of symptomatic malaria in the DRC as DHS surveys do not sample health facilities.

\section{CONCLUSION}

This study evaluated risk factors for $P$. falciparum infection among adults in the DRC using data from the nationally representative, population-based DHS survey. Overall prevalence of infection was high, $30 \%$, and grossly unchanged from the prior 2007 survey. We observed high spatial heterogeneity across the country and identified individual, household and community-level risk factors for malaria such as male sex, traditional housing and decreasing within household net coverage. These findings support the need for sustained investment in malaria control in the DRC and can be used to develop targeted interventions with maximal impact.

\section{Author affiliations}

${ }^{1}$ Department of Epidemiology, Gillings School of Global Public Health, University of North Carolina at Chapel Hill, Chapel Hill, North Carolina, USA

${ }^{2}$ Division of Infectious Diseases, Department of Medicine, School of Medicine, University of North Carolina at Chapel Hill, Chapel Hill, North Carolina, USA ${ }^{3}$ National AIDS Control Program, Kinshasa, Congo (the Democratic Republic) ${ }^{4}$ School of Public Health, University of Kinshasa Faculty of Medicine, Kinshasa, Congo (the Democratic Republic)

${ }^{5}$ Department of Pathology and Laboratory Medicine, Brown University Warren Alpert Medical School, Providence, Rhode Island, USA

${ }^{6}$ Medical Research Council Centre for Global Infectious Disease Analysis, Department of Infectious Disease Epidemiology, Imperial College London, London, UK

${ }^{7}$ Department of Geography, University of North Carolina at Chapel Hill, Chapel Hill, North Carolina, USA

${ }^{8}$ Curriculum in Genetics and Molecular Biology, University of North Carolina at Chapel Hill, Chapel Hill, North Carolina, USA

Acknowledgements The authors would like to thank all the study participants and all study team members. The authors would also like to thank Stephanie Doctor for assistance with sample processing.

Contributors MDF conducted the analyses and drafted the manuscript. NFB provided assistance with data management and the risk factor analyses. JBP and KLT conducted the PCR assays. JM supervised blood spot processing. MK and ATK oversaw sample management. $\mathrm{OA}$ and JAB generated and processed the sequencing data. JKE, RV, ME and EWG provided guidance with the study design and provided analytical assistance. JJJ and SRM conceived of the study and oversaw study set-up. All authors edited the manuscript and approved the final draft.

Funding This study was supported by R01Al107949 to SRM and T32AI070114 to MDF from the National Institutes of Health National Institute for Allergy and Infectious Disease. This study was also supported by a Burroughs Wellcome Fund-American Society for Tropical Medicine and Hygiene fellowship to JBP and R01Al139520 to JAB. RV is jointly funded by the UK Medical Research Council (MRC) and the UK Department for International Development (DFID) under the MRC/ DFID Concordat agreement and is also part of the EDCTP2 programme supported by the European Union.

Competing interests JBP reports support from the WHO; JPB and SRM report non-financial support from Abbott Laboratories, which has performed laboratory testing in-kind as part of their hepatitis research, outside the submitted work.

Patient and public involvement Patients and/or the public were not involved in the design, or conduct, or reporting or dissemination plans of this research.

Patient consent for publication Not required.

Ethics approval This study was approved by the Internal Review Board at The University of North Carolina, Chapel Hill and at the Kinshasa School of Public Health. Informed consent was obtained from each individual age 18 or older, or from a parent or legal guardian for children and adolescents under age 18.

Provenance and peer review Not commissioned; externally peer reviewed.

Data availability statement Data are available in a public, open access repository and upon request. Data used in this study from the Demographic Health Surveys Program are available upon request at (https://dhsprogram.com/what-we-do/ 
survey/survey-display-421.cfm). Raw sequencing data are publicly available through the NCBI SRA (accession number: PRJNA545347). Molecular data included in this study are available from the corresponding author upon reasonable request.

Open access This is an open access article distributed in accordance with the Creative Commons Attribution Non Commercial (CC BY-NC 4.0) license, which permits others to distribute, remix, adapt, build upon this work non-commercially, and license their derivative works on different terms, provided the original work is properly cited, appropriate credit is given, any changes made indicated, and the use is non-commercial. See: http://creativecommons.org/licenses/by-nc/4.0/.

ORCID iD

Molly Deutsch-Feldman http://orcid.org/0000-0001-6191-930X

\section{REFERENCES}

1 The World Health Organization. World malaria report 2019. Geneva, Switzerland: The World Health Organization, 2019.

2 Verity R, Aydemir O, Brazeau NF, et al. The impact of antimalarial resistance on the genetic structure of Plasmodium falciparum in the DRC. bioRxiv 2019;656561.

3 Verity R, Hathaway NJ, Waltmann A, et al. Plasmodium falciparum genetic variation of VAR2CSA in the Democratic Republic of the Congo. Malar J 2018:17:1-8.

4 Taylor SM, Messina JP, Hand CC, et al. Molecular malaria epidemiology: mapping and burden estimates for the Democratic Republic of the Congo, 2007. PLoS One 2011;6:e16420.

5 The World Health Organization. Key points: world malaria report 2017. The World Health Organization, 2017.

6 Jenkins R, Omollo R, Ongecha M, et al. Prevalence of malaria parasites in adults and its determinants in malaria endemic area of Kisumu County, Kenya. Malar J 2015;14:263.

7 Lindblade KA, Steinhardt L, Samuels A, et al. The silent threat: asymptomatic parasitemia and malaria transmission. Expert Rev Anti Infect Ther 2013;11:623-39.

8 Lin JT, Saunders DL, Meshnick SR. The role of submicroscopic parasitemia in malaria transmission: what is the evidence? Trends Parasitol 2014;30:183-90.

9 Levitz L, Janko M, Mwandagalirwa K, et al. Effect of individual and community-level bed net usage on malaria prevalence among underfives in the Democratic Republic of Congo. Malar J 2018;17:39.

10 Janko MM, Irish SR, Reich BJ, et al. The links between agriculture, Anopheles mosquitoes, and malaria risk in children younger than 5 years in the Democratic Republic of the Congo: a population-based, cross-sectional, spatial study. Lancet Planet Health 2018;2:e74-82.

11 Ferrari G, Ntuku HMT, Ross A, et al. Identifying risk factors for Plasmodium infection and anaemia in Kinshasa, Democratic Republic of Congo. Malar J 2016:15:362.

12 Ngatu NR, Kanbara S, Renzaho A, et al. Environmental and sociodemographic factors associated with household malaria burden in the Congo. Malar J 2019;18:53.

13 Messina JP, Taylor SM, Meshnick SR, et al. Population, behavioural and environmental drivers of malaria prevalence in the Democratic Republic of Congo. Malar J 2011:10:161

14 Aydemir O, Janko M, Hathaway NJ, et al. Drug-Resistance and population structure of Plasmodium falciparum across the Democratic Republic of Congo using high-throughput molecular inversion probes. $J$ Infect Dis 2018;218:946-55.

15 Deutsch-Feldman M, Aydemir O, Carrel M, et al. The changing landscape of Plasmodium falciparum drug resistance in the Democratic Republic of Congo. BMC Infect Dis 2019;19:872

16 Wilson ML, Krogstad DJ, Arinaitwe E, et al. Urban malaria: understanding its epidemiology, ecology, and transmission across seven diverse ICEMR network sites. Am J Trop Med Hyg 2015;93:110-23.

17 Tatem AJ, Gething PW, Smith DL, et al. Urbanization and the global malaria recession. Malar J 2013:12:133.

18 Hay SI, Guerra CA, Tatem AJ, et al. Urbanization, malaria transmission and disease burden in Africa. Nat Rev Microbiol 2005;3:81-90.

19 Alirol E, Getaz L, Stoll B, et al. Urbanisation and infectious diseases in a globalised world. Lancet Infect Dis 2011;11:131-41.

20 Donnelly MJ, McCall PJ, Lengeler C, et al. Malaria and urbanization in sub-Saharan Africa. Malar J 2005;4:12.

21 Andrada A, Herrera S, Inyang U, et al. A subnational profiling analysis reveals regional differences as the main predictor of ITN ownership and use in Nigeria. Malar J 2019;18:185.

22 Tokponnon FT, Aholoukpe B, Denon EY, et al. Evaluation of the coverage and effective use rate of long-lasting insecticidal nets after nation-wide scale up of their distribution in Benin. Parasit Vectors 2013;6:265

23 Ladi-Akinyemi T, Ladi-Akinyemi B, Olatona F, et al. Ownership and utilization of long-lasting insecticide nets among caregivers of children under- 5 years in Ogun state, Nigeria: a rural-urban comparison. J Clin Sci 2018;15:145.

24 Stebbins RC, Emch M, Meshnick SR. The effectiveness of community bed net use on malaria parasitemia among children less than 5 years old in Liberia. Am J Trop Med Hyg 2018;98:660-6.

25 Djamouko-Djonkam L, Mounchili-Ndam S, Kala-Chouakeu N, et al. Spatial distribution of Anopheles gambiae sensu lato larvae in the urban environment of Yaoundé, Cameroon. Infect Dis Poverty 2019;8:84.

26 Demographic and Health Surveys. Team and partners. Available: https://dhsprogram.com/Who-We-Are/About-Us.cfm

27 Ministère du Plan and Macro International. Enquête Démographique et de Santé, République Démocratique Du Congo 2007. Calverton, MD, USA: Ministère du Plan and Macro International, 2008.

28 Vassen M, Thiam M, Le T. The Demographic and Health Surveys. In: Household sample surveys in developing and transition countries. New York, NY: United Nations Publishing, 2005.

29 Doctor SM, Liu Y, Whitesell A, et al. Malaria surveillance in the Democratic Republic of the Congo: comparison of microscopy, PCR, and rapid diagnostic test. Diagn Microbiol Infect Dis 2016;85:16-18.

30 Rutstein SO. The DHS wealth index: approaches for rural and urban areas, 2008.

31 Tusting LS, Bottomley C, Gibson H, et al. Housing improvements and malaria risk in sub-Saharan Africa: a Multi-Country analysis of survey data. PLoS Med 2017;14:e1002234.

32 Lindsay SW, Jawara M, Mwesigwa J, et al. Reduced mosquito survival in metal-roof houses may contribute to a decline in malaria transmission in sub-Saharan Africa. Sci Rep 2019;9:7770.

33 Mayala B, Fish TD, Eitelberg D, et al. The Geospatial covariate datasets manual. Rockville, Maryland: The DHS Program, ICF, 2018.

34 Plowe CV, Djimde A, Bouare M, et al. Pyrimethamine and proguanil resistance-conferring mutations in Plasmodium falciparum dihydrofolate reductase: polymerase chain reaction methods for surveillance in Africa. Am J Trop Med Hyg 1995;52:565-8.

35 Beshir KB, Hallett RL, Eziefula AC, et al. Measuring the efficacy of anti-malarial drugs in vivo: quantitative PCR measurement of parasite clearance. Malar J 2010;9:312.

36 Tangpukdee N, Duangdee C, Wilairatana P, et al. Malaria diagnosis: a brief review. Korean J Parasitol 2009;47:93-102.

37 Mwandagalirwa MK, Levitz L, Thwai KL, et al. Individual and household characteristics of persons with Plasmodium falciparum malaria in sites with varying endemicities in Kinshasa Province, Democratic Republic of the Congo. Malar J 2017;16:456.

38 Hyde JE. Drug-resistant malaria - an insight. Febs J 2007:274:4688-898.

39 Antonia AL, Taylor SM, Janko M, et al. A cross-sectional survey of Plasmodium falciparum PfCRT mutant haplotypes in the Democratic Republic of Congo. Am J Trop Med Hyg 2014;90:1094-7.

40 Taylor SM, Antonia AL, Parobek CM, et al. Plasmodium falciparum sulfadoxine resistance is geographically and genetically clustered within the Dr Congo. Sci Rep 2013;3:9-11.

41 Giorgi E, Diggle PJ. PrevMap: An $R$ Package for Prevalence Mapping. J Stat Softw 2017;78.

42 Marivoet W, De Herdt T. Tracing down real socio-economic trends from household data with erratic sampling frames: the case of the Democratic Republic of the Congo. J Asian Afr Stud 2017;53.

43 Noor AM, Alegana VA, Gething PW, et al. Using remotely sensed night-time light as a proxy for poverty in Africa. Popul Health Metr 2008;6:5

44 Wesolowski A, Eagle N, Tatem AJ, et al. Quantifying the impact of human mobility on malaria. Science 2012;338:267-70.

45 WorldPop. Africa Continental Population Datasets (2000 - 2020), 2016. Available: https://www.worldpop.org/doi/10.5258/SOTON/ WP00013

46 Harris I, Jones PD, Osborn TJ, et al. Updated high-resolution grids of monthly climatic observations - the CRU TS3.10 dataset. Int J Climatol 2014;43:623-42.

47 Weiss DJ, Nelson A, Gibson HS, et al. A global map of travel time to cities to assess inequalities in accessibility in 2015. Nature 2018;553:333-6.

48 R Core Team. R: a language and environment for statistical computing. Vienna, Austria: R Foundation for Statistical Computing, 2017. https://www.r-project.org/

49 Lumley T. Analysis of complex survey samples. J Stat Softw 2004;9:1-19.

50 Lumley T. Survey: analysis of complex survey samples. R Package v 3, 2019: 35-1. 
51 Westreich D, Greenland S. The table 2 fallacy: presenting and interpreting confounder and modifier coefficients. Am J Epidemiol 2013;177:292-8.

52 Croft T, Marhsall A, Allen C. Guide to DHS statistics. Rockville, Maryland: ICF, 2018.

53 The Demographic and Health Survey Program. Demographic and health survey (DRC-DHS II) supplemental malaria report. The Demographic and Health Survey, 2015.

54 The World Health Organization. Democratic Republic of the Congo, 2016. Available: http://www.who.int/malaria/publications/countryprofiles/profile_cod_en.pdf?ua=1 [Accessed 21 Jun 2018].

55 Essendi WM, Vardo-Zalik AM, Lo E, et al. Epidemiological risk factors for clinical malaria infection in the highlands of Western Kenya. Malar J 2019;18:211.

56 Human Factors and Malaria, 2018. Centers for Disease Controland Prevention. Available: https://www.cdc.gov/malaria/about/biology/ index.html [Accessed 10/15/2018].

57 Ippolito MM, Searle KM, Hamapumbu H, et al. House Structure Is Associated with Plasmodium falciparum Infection in a LowTransmission Setting in Southern Zambia. Am J Trop Med Hyg 2017;97:1561-7.

58 The World Health Organization. Achieving and maintaining universal coverage with long-lasting insecticidal nets for malaria control. Geneva, Switzerland: The World Health Organization, 2017.

59 Yang G-G, Kim D, Pham A, et al. A meta-regression analysis of the effectiveness of mosquito nets for malaria control: the value of longlasting insecticide nets. Int J Environ Res Public Health 2018;15. doi:10.3390/ijerph15030546. [Epub ahead of print: 19 Mar 2018]

60 Dambach P, Schleicher M, Korir P, et al. Nightly biting cycles of Anopheles species in rural northwestern Burkina Faso. J Med Entomol 2018;55:1027-34.
61 Thomsen EK, Koimbu G, Pulford J, et al. Mosquito behavior change after distribution of bednets results in decreased protection against malaria exposure. J Infect Dis 2017;215:790-7.

62 Rae JD, Nosten S, Proux S, et al. The role of monitoring and evaluation to ensure functional access to community-based early diagnosis and treatment in a malaria elimination programme in eastern Myanmar. Malar J 2019;18:50.

63 Eisele TP. Mass drug administration can be a valuable addition to the malaria elimination toolbox. Malar J 2019;18:281.

64 Landier J, Parker DM, Thu AM, et al. Effect of generalised access to early diagnosis and treatment and targeted mass drug administration on Plasmodium falciparum malaria in eastern Myanmar: an observational study of a regional elimination programme. Lancet 2018;391:1916-26.

65 Romay-Barja M, Jarrin I, Ncogo P, et al. Rural-Urban differences in household treatment-seeking behaviour for suspected malaria in children at Bata district, equatorial guinea. PLoS One 2015;10:e0135887.

66 Keiser J, SINGER BH, SMITH TA, et al. Urbanization in Sub-Saharan Africa and Implication for Malaria Control. In: Berman J, Alilio MS, Mills A, et al, eds. The Intolerable Burden of Malaria II: What's New, What's NeededBethesda Maryland, 2004: 118-27.

67 Ministere du Plan et Suivi de la Mise en oeuvre de la Revolution de la Modernite - MPSMRM/Congo, Ministere de la Sante Publique - MSP/Congo, ICF International. Republique Democratique Du Congo Enquete Demographique et de Sante (EDS-RDC) 2013-2014. Rockville, Maryland, USA: MPSMRM, MSP, and ICF International, 2014. http://dhsprogram.com/pubs/pdf/FR300/ FR300.pdf 